\title{
Improvements in coded aperture thermal neutron imaging
}

\author{
Peter E. Vanier \\ Brookhaven National Laboratory, NNS Dept., Building 197C, Upton, NY 11973
}

\begin{abstract}
A new thermal neutron imaging system has been constructed, based on a $20-\mathrm{cm} \times 17-\mathrm{cm} \mathrm{He}-3$ position-sensitive detector with spatial resolution better than $1 \mathrm{~mm}$. New compact custom-designed position-decoding electronics are employed, as well as high-precision cadmium masks with Modified Uniformly Redundant Array patterns. Fast Fourier Transform algorithms are incorporated into the deconvolution software to provide rapid conversion of shadowgrams into real images. The system demonstrates the principles for locating sources of thermal neutrons by a stand-off technique, as well as visualizing the shapes of nearby sources. The data acquisition time could potentially be reduced two orders of magnitude by building larger detectors.
\end{abstract}

Keywords: Neutron, image, coded aperture, MURA, FFT, He-3, ionization chamber, position-sensitive

\section{INTRODUCTION}

Two-dimensional position-sensitive He-3 ionization chambers ${ }^{1}$ have been developed at BNL over many years for use in neutron diffraction experiments at the High Flux Beam Reactor (now shut down) and are now used at several other research facilities. Cold neutrons were particularly useful in protein crystallography, because they scattered most efficiently from hydrogen, whereas $x$-rays emphasized higher- $Z$ atoms in a molecular structure. Thus the structural information obtained using neutrons was complementary to that generated with $\mathrm{x}$-rays at the National Synchrotron Light Source. The He-3 ionization chambers were designed to have high detection efficiency for thermal neutrons, spatial resolution better than $1 \mathrm{~mm}$, a high degree of spatial linearity, and excellent stability over long periods of time. The same characteristics required for crystallography are also essential for imaging.

Coded aperture imaging ${ }^{2}$, which is an extension of pinhole camera photography, employs masks containing multiple apertures with different pixel shapes arranged in unique patterns. The dimensions of the individual pixel apertures determine the resolution of the image, while the total area of the apertures determines the speed with which the image can be acquired. Many designs of coded aperture masks have been created for $\mathrm{x}$-ray imaging of nuclear explosions and astronomical objects. Among the most successful are Uniformly Redundant Arrays (URA.s) ${ }^{3}$ and Modified Uniformly Redundant Arrays (MURAs), which are widely used in X-ray and gamma ray astronomy. In the early 1990's, KlausPeter Ziock of LLNL built a gamma-ray imaging system ${ }^{5}$ using an URA that could be used for counting warheads on a re-entry vehicle in confirmation of potential arms control agreements. (There are now commercial gamma cameras available that use coded apertures). Subsequently, Leon Forman of BNL recognized that an analogous instrument could be devised to create images using thermal neutrons ${ }^{6}$.

In 1995, initial experiments were performed to show that thermal neutrons from a Cf-252 source surrounded by a few centimeters of polyethylene could cast a shadow of a cadmium slit on a He-3 tube, with a contrast ratio of about 3 to 1 . This ratio represented the proportion of detected neutrons useful for forming an image. That is, of every 4 neutrons detected, 3 of them traveled about 3 meters directly from the thermalizing material to the detector, passed through the aperture in the cadmium mask and had low enough energy to react with the He-3. The remaining 1 out of 4 neutrons produced a uniform background, unrelated to the source location. This uniform illumination is partly from neutrons randomly scattered (in the air or other surroundings) and then reaching the detector at oblique angles, and partly from epithermal neutrons with energy high enough to pass through the cadmium but low enough to be absorbed by He-3. A crude version of a coded aperture neutron camera was demonstrated using an array of position-sensitive He-3 tubes and a $3 \times 5$ URA mask ${ }^{6}$. This device suffered from non-linearity in the encoding of position, poor spatial resolution, and large variations in sensitivity as a function of position in the image plane. 
In 1996, a high-resolution position-sensitive He-3 proportional counter was used in a Cd-lined box to construct a codedaperture imaging device with an $11 \times 13$ URA mask ${ }^{7}$. This imager was shown to be capable of locating a thermal neutron source within one pixel at distances up to 65 meters. Later, a $29 \times 31$ URA mask was employed ${ }^{7,8}$, not only to locate a "point" source at ranges up to $60 \mathrm{~m}$, but also to image more complex shapes at $3 \mathrm{~m}$ range, including as many as 6 separated sources.

In this paper, a number of improvements of the imager will be described, although the basic He- 3 wire chamber remains unchanged. The wooden $\mathrm{Cd}$-lined box was replaced by an $\mathrm{Al}$ box, equipped with sliding supports for the mask at various focal lengths. The new construction provides better dimensional stability, and allows reproducible alignment of the mask with the detector elements. New high-precision MURA masks were fabricated to our specifications by Max Levy Autograph, Inc., and the data analysis software was modified to analyze the shadowgrams using fast Fourier transforms ${ }^{9}$ (FFTs). In addition, the position-encoding electronics have been rebuilt by BNL's Instrumentation Division in the form of a single board that replaces two racks of NIM modules and a CAMAC crate.

\section{HARDWARE}

The newly reconstructed thermal neutron imager is shown in Fig. 1. The basis of this instrument is a 2-dimensional $20 \mathrm{~cm} \times 17 \mathrm{~cm}$ position-sensitive proportional counter (1) containing about 6 atmospheres of He-3. A thermal neutron reacts with $\mathrm{He}-3$ to form a proton and a triton with $768 \mathrm{keV}$ kinetic energy available to ionize electrons in the gas. In the proportional mode, the applied bias field is sufficient to cause some electron multiplication. The motion of the ionized electrons in the bias field is detected by two perpendicular planar arrays of cathode strips etched on a printed circuit board and connected to charge-sensitive preamplifiers at a number of resistively coupled nodes. The center of charge in each direction is determined to within $1 \mathrm{~mm}$ by resistive charge division between the three preamplifier nodes closest to the event. All of the position-encoding electronics required to create a digital histogram are now contained in a single custom board (2). The histogram can be initialized or interrogated, when required, by a computer (3) that performs further analysis of the data. The shadows are cast on the front of the detector by a coded aperture (4) constructed from $\mathrm{Cd}$ sheet glued to an $\mathrm{Al}$ substrate. The other 5 sides of the detector are shielded from scattered neutrons by a Cd-lined box equipped with 4 support frames on which the mask can be clipped at different distances from the face of the detector.
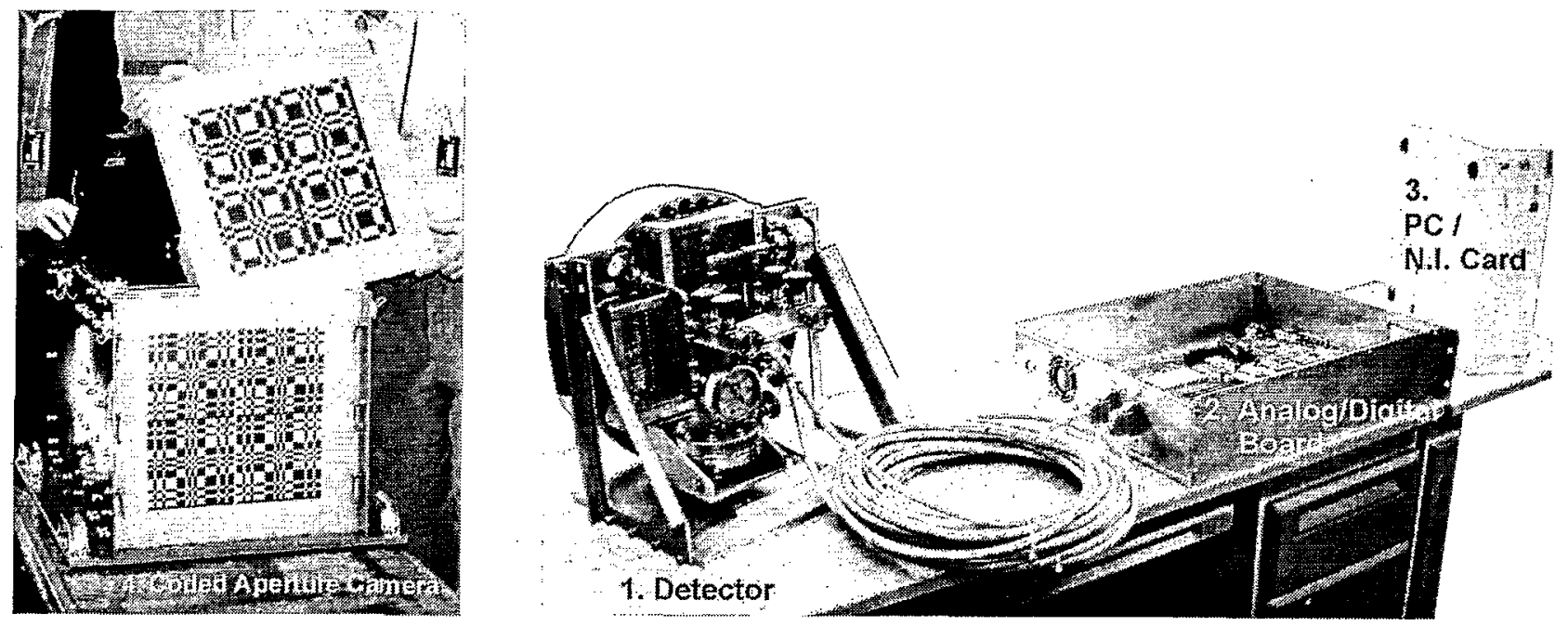

Figure 1: Components of the thermal neutron imaging system.

Most neutron detectors that are not designed for imaging would include Cd-clad plastic thermalizers surrounding a set of He-3 tubes, so as to eliminate the scattered thermal neutrons while detecting only the fast and epithermal neutrons. The $\mathrm{BNL}$ imager has no thermalizer other than the He-3 itself, and relies on thermalization to take place in the objects in the 
scene. Thus this measurement makes use of a population of neutrons that is usually neglected. The tests described in this paper were performed using an Am-241/Be source inserted into a $6 \mathrm{~cm}$-dia. cylindrical space on the axis of a $45 \mathrm{~cm}$ dia. cylindrical paraffin moderator. The source cavity was plugged with a paraffin rod, slightly less than $6 \mathrm{~cm}$ in diameter, and a $15-\mathrm{cm}$ dia. circular region of the end of the moderator was exposed to the camera. All other surfaces of the moderator were surrounded with boron-loaded plastic shielding. Simple geometric shapes were framed on the face with Cd sheet, so as to create multi-pixel targets to be detected in the images.

Two of the new MURA coded aperture masks are shown in Fig.1, with dimensions $19 \times 19$ and 31 x 31 pixels. A high resolution mask with dimensions $47 \times 47$ pixels (not shown) was also fabricated and tested. All three masks were chosen to be antisymmetric.

\section{SOFTWARE}

Data acquisition and analysis is performed using "virtual instrument" programs constructed with the LabView ${ }^{\mathrm{TM}}$ development system from National Instruments. Two-dimensional histograms are input on a 32-bit parallel digital I/O card from National Instruments and stored as $1.2 \mathrm{Mb}$ shadowgram data files consisting of an unsigned integer array of approximately $500 \times 600$ data values. Other analysis programs can read and process these files while the data acquisition card continues to accumulate more data.

The basic algorithm for recovering a real image from a MURA shadowgram has been described in the literature ${ }^{4}$, and on websites discussing coded aperture imaging ${ }^{2}$. It requires constructing a matrix $\mathrm{G}$ in which each cell that corresponds to an open pixel of the mask is given a value +1 and those cells representing closed pixels of Cd are given a value -1 . Suppose there were a distant point source on the axis of the camera. One can identify the data values for areas of the detector that would be directly illuminated through the mask and sum them, then subtract the total counts falling in areas covered by $\mathrm{Cd}$. The resulting number of counts is assigned to the pixel at the center of the image array. The intensities for other pixels in the image are evaluated by offsetting the G-matrix step by step relative to the measured array of data. The complete calculation amounts to a two-dimensional correlation between the mask pattern and the shadowgram array. One reason this process generates a unique image is that the correlation of a MURA pattern with itself is a twodimensional delta-function. In other words, two identical MURA masks placed together will give a constant transmission factor (1/4) for all vertical and horizontal offsets other than perfect alignment, when the transmission is $1 / 2$. For large arrays, if performed by nested loops of multiplications and summations, the number of operations needed to generate the image grows as the square of the number of pixels.

A more efficient method of reconstructing the image is to generate digital fast Fourier transforms of the data array and the mask pattern. A single scalar multiplication of the matrix elements is then performed, and the inverse Fourier transform of the product generates the image. The number of operations in this calculation increases only linearly with the number of pixels, and the results are almost identical to those obtained via the traditional calculation. Since LabView ${ }^{\mathrm{TM}}$ contains FFTs in its digital signal-processing library, the programming is quite simple.

When the raw data values are binned into pixels matching the mask, there are arbitrary choices of $x$ and $y$ offsets that can affect the image quality by arranging for a bright spot in the scene to line up with a single mask pixel or to be shared between 2 or 4 pixels. The arbitrariness of this alignment can be avoided somewhat by subdividing the mask pixels into smaller areas and calculating the FFT image based on smaller intervals ${ }^{9}$ in $\mathrm{x}$ and $\mathrm{y}$. The limiting case is reached when the unit area used for the calculation is the size of the original data pixels. Performing the deconvolution in this way increases the time for the calculation, but provides a smoother image than is obtained by initially binning the data into mask-sized pixels. Various compromises can be found between speed of calculation and the degree of aliasing of the image by binning the data into areas $1 / 4,1 / 9$, or $1 / 16$ of the mask pixels, or by not binning at all.

\section{UNIFORMITY CORRECTIONS}

The detector was uniformly illuminated with an extended source, approximately $15 \mathrm{~cm}$ in diameter at a distance of 180 $\mathrm{cm}$ for a time sufficient to accumulate at least 1 million total counts. The typical intensity map, shown in Figure 2 illustrates the variations in efficiency of the detector as a function of position. No corrections are required for linearity 
of the spatial mapping, because the locations of events are determined by the fixed positions of precisely constructed wires. However, some periodic variations in intensity are seen in both $\mathrm{x}$ and $\mathrm{y}$ directions, resulting from differences in the probability of detection as a function of the distance of a primary event to the nearest anode wire and the distance to the nearest node of the arrays of preamplifiers. Thus the intensity map shows a grid pattern that resembles the wire arrays themselves. By summing the rows and columns of this data file, one can plot the average response as a function of $x$ and $y$, as shown in Figure 3. The long wavelength oscillations in both $x$ and $y$ directions peak at the nodes where preamplifiers are connected. The short wavelength oscillations in the y direction peak at the actual anode wires where the electron showers are collected. The fine striations are only seen running horizontally, because the anode wires are horizontal.

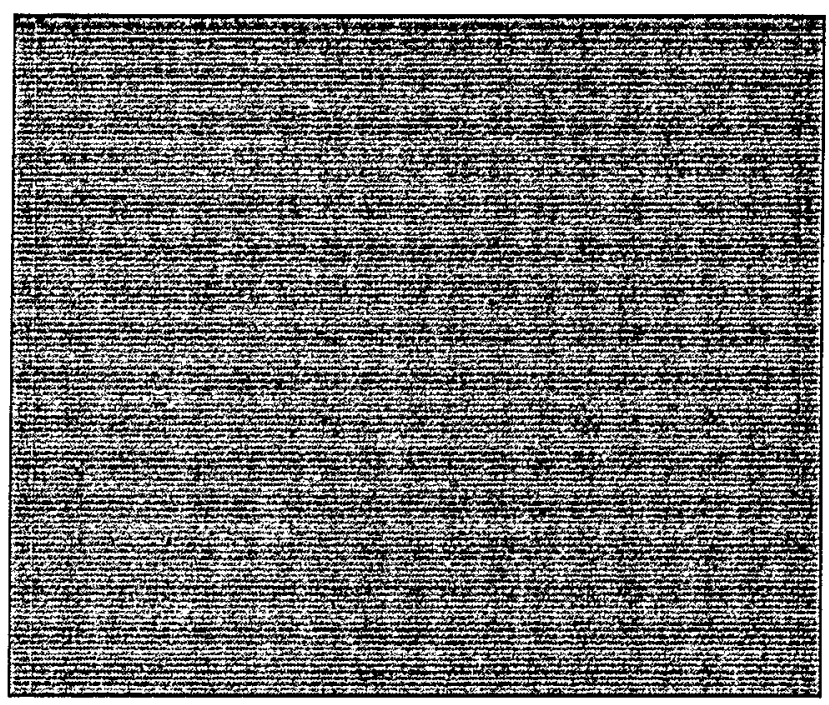

Figure 2: Variations in efficiency of the He-3 detector as a function of position under uniform illumination.

These average fluctuations in response are remarkably stable and reproducible, provided the fill pressure and bias voltages are kept constant. Therefore, one can make a correction to a raw data file using the row and column averages from uniform illumination to normalize the response as a function of position. (Normalizing each pixel on the basis of its individual response would require very long exposures in order to obtain good counting statistics in each pixel). The row and column normalization procedure removes much of the appearance of the wire array, leaving mainly statistical fluctuations. The same normalization can be applied to a shadowgram when the mask is in place, as will be seen later.

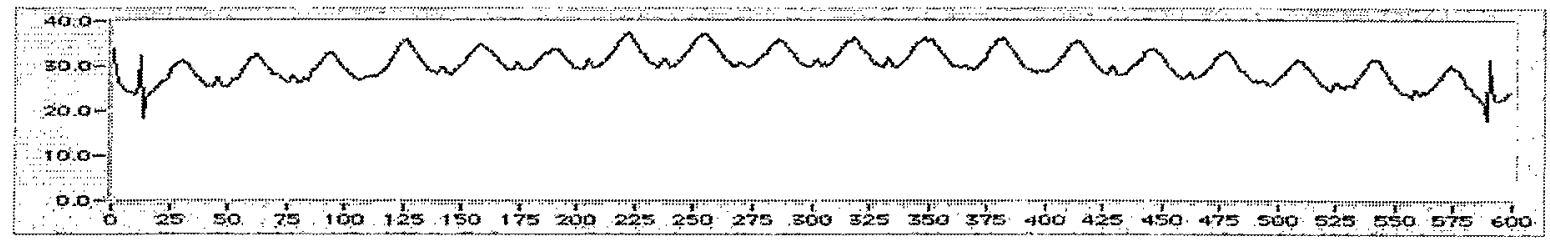

Column number

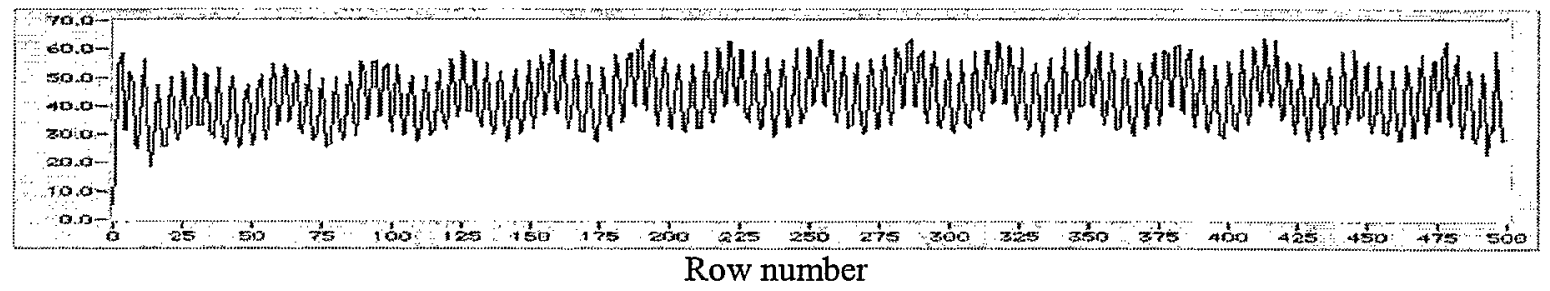

Figure 3: Row and column summations of uniformly illuminated image. 


\section{SHADOWGRAMS AND IMAGES}

\subsection{Near-field limitations}

For a point source on the axis, if the object range, $r$ is much greater than the focal length, $f$, the shadow of the mask resembles the mask, but with a magnification slightly greater than unity. The processing algorithm takes account of the fact that the shadow of each pixel is slightly larger than the mask pixel itself. The magnitude of the correction is based on an assumed value of the range, which is an input to the calculation. However, if the object is too close to the imaging system, the projection of the primitive mask pattern on the detector is larger than the sensitive area, and insufficient data are collected to generate a unique image. The present set of masks was chosen to have a $15-\mathrm{cm} \times 15-\mathrm{cm}$ primitive pattern, tiled in a $2 \times 2$ mosaic. Since the detector sensitive area is $20 \times 17 \mathrm{~cm}$, the maximum magnification,

$$
m_{\max }={ }^{17} / 15=1+f / r_{\min } \text {. }
$$

Therefore, for this detector and masks, the minimum range is

For a $20 \mathrm{~cm}$ focal length, the closest imaging range is $150 \mathrm{~cm}$.

$$
r_{\min }=7.5 f
$$

The usable magnification is physically limited by the uncertainty in position of an event in the detector when the thermal neutron enters at a steep angle to the optical axis. The mean free path of neutrons at room temperature $(0.025 \mathrm{eV}$ kinetic energy) is about $5 \mathrm{~mm}$ in He-3 at $6 \mathrm{~atm}$. Therefore, a neutron entering the detector at an angle of $30^{\circ}$ from the axial direction will be detected at some position with an uncertainty of about $2.5 \mathrm{~mm}$. This effect would limit the use of a high resolution mask ( $3 \mathrm{~mm}$ per pixel) at short ranges. However, the lower resolution mask ( $8 \mathrm{~mm}$ per pixel) can still provide an image. Since the Cd mask is quite thin, it is not a limiting factor on angular aperture, as is the case with gamma cameras with $\mathrm{cm}$-thick masks.

\subsection{Minimal focal length}

Using the shortest available focal length, where the mask is placed $2 \mathrm{~cm}$ from the plane where thermal neutrons can first interact with $\mathrm{He}-3$, it is possible to test the image-forming algorithm under the most favorable conditions. An extended source at a relatively short range creates a sharp shadow of the mask with little magnification or parallax, and with good statistics. A shadowgram formed in this way using the $19 \times 19$ mask is shown in Figure 4a. When the corrections for detector sensitivity are applied, the shadow appears as in Figure $4 \mathrm{~b}$. The converted image, shown in Figure 5a, is an empirically determined point-spread distribution function for the system. The excess counts representing the source accumulate in a single pixel just as they would with a point source at a long distance. A similar process was used to obtain the image in Figure $5 b$ with a 47 x 47-pixel MURA mask.

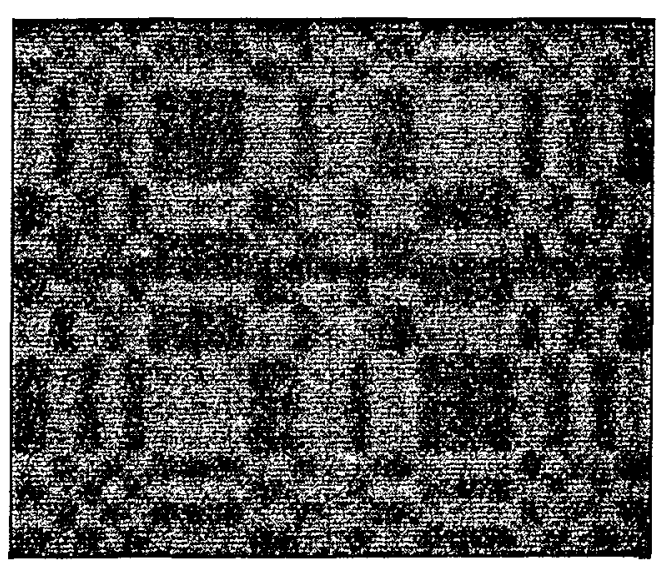

(a)

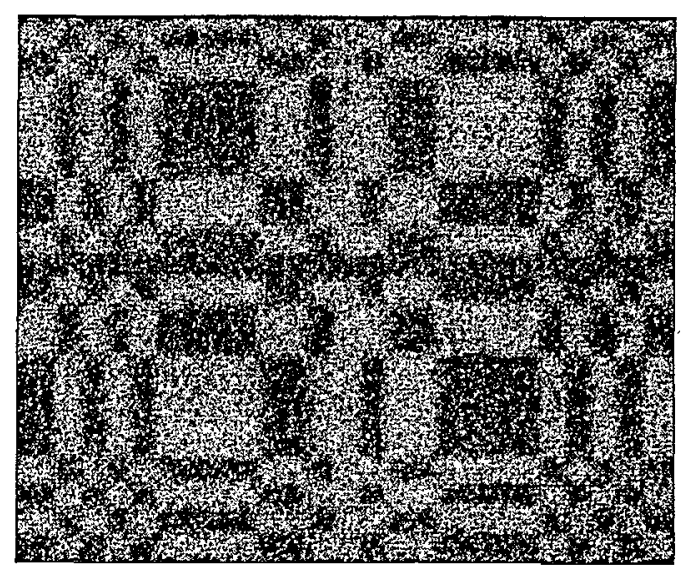

(b)

Figure 4: Shadowgram of $19 \times 19$ mask on the face of the detector (a) raw data and

(b) data corrected for detector sensitivity by row and column averages of a uniformly illuminated detector. 


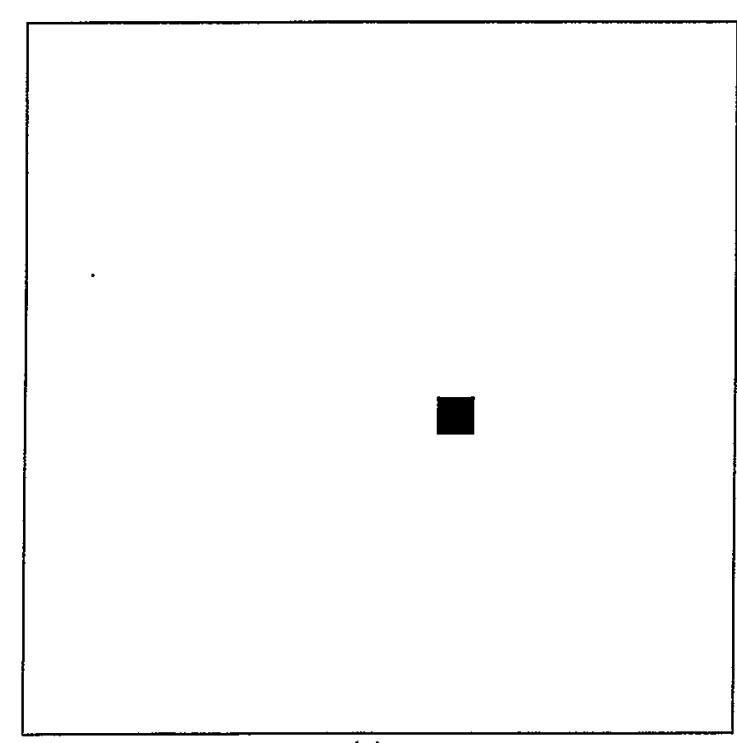

(a)

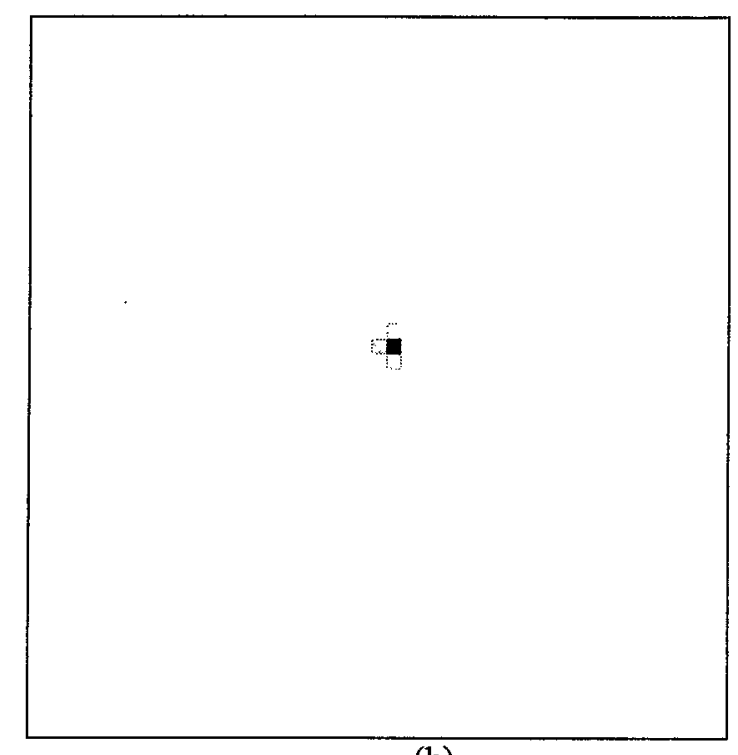

(b)

Figure 5: Images calculated from short focal-length data by FFT method using (a) $19 \times 19$ MURA mask, and (b) $47 \times 47$ MURA mask.

\subsection{Extended sources}

Using a $47 \times 47$ pixel mask, located at a focal length of $20 \mathrm{~cm}$, an image was formed of an extended polyethylene object framed by cadmium sheets arranged in the shape of a right triangle with $10-\mathrm{cm}$ vertical and horizontal sides at a range of $1.8 \mathrm{~m}$. The resulting image is shown in Figure 6a, which was calculated by the method in which data are first binned into areas the size of the mask pixels. The "staircase" or aliasing effect on diagonal lines is apparent, and the intensities of individual object-related pixels can vary considerably with the arbitrary choice of zero-offsets used to select a portion of the shadowgram from the raw data. This method has the advantage of speed of computation, but requires some operator: judgement in order to find the "best picture".

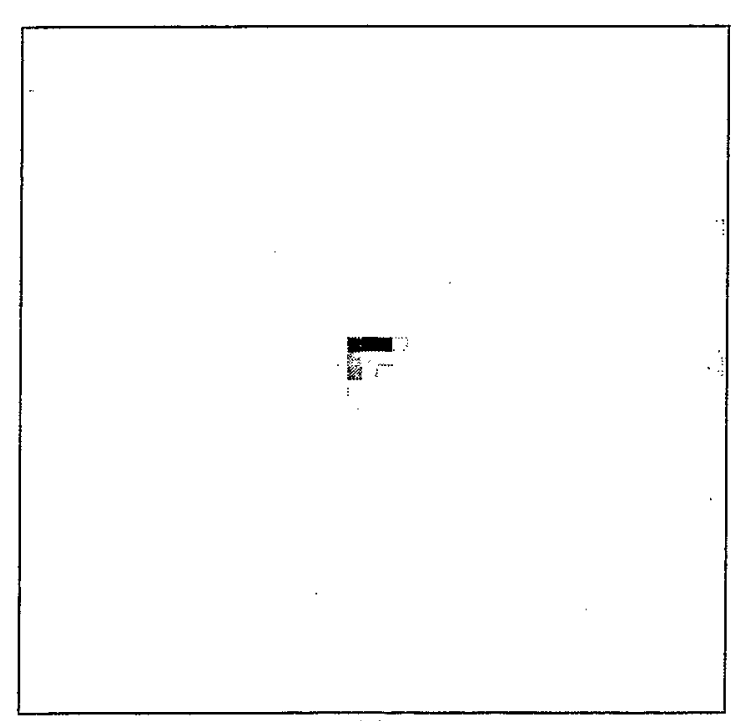

(a)

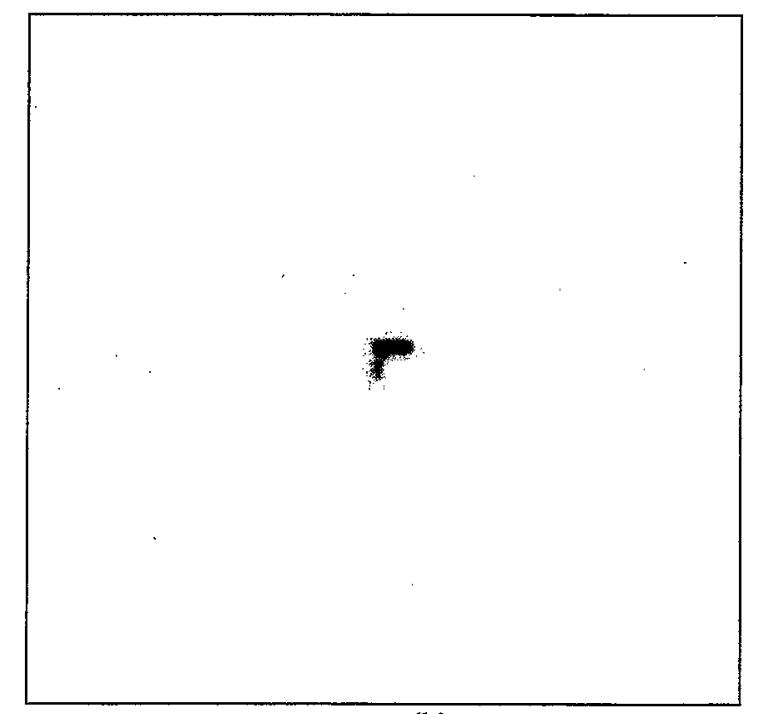

(b)

Figure 6: Image of triangular-framed area of polyethylene moderator, (a) data binned to match areas of mask pixels and (b) intensity calculated for each unbinned data value 
An alternative way to process the image is shown in Figure $6 \mathrm{~b}$, where individual data values have been used independently without prior binning. In order to perform the FFT, the array representing the mask was expanded by inserting duplicate columns and rows so that each mask array element would correspond to an element in the raw data file. Processing on this fine a scale has the disadvantage of a larger number of multiplication operations, as well as displaying the high-frequency statistical noise that would be averaged by binning. However, it removes much of the staircasing and the effects of arbitrary offsets in the final image. The reason that the triangle did not exhibit the expected uniform intensity became apparent in subsequent experiments.

Figure 7 shows images acquired of the same cylindrical moderator viewed on its axis, outlined by (a) a 10-cm square cadmium frame and (b) the $15-\mathrm{cm}$ dia. circular borated plastic shield. The shapes of the outlines are evident in the images as expected, but an additional feature systematically appears that was not expected. The central circular region of each image exhibits a lower thermal neutron flux than the surrounding brighter ring. The brightest circular contour is consistent in size with the 6-cm dia. tube containing the source. Further experiments will be needed to help explain the observed intensity distribution. It appears that neutrons which leak through the annular space around the loosely-fitting central paraffin plug make a strong contribution to the image, compared to those that diffuse through tens of $\mathrm{cm}$ of paraffin.

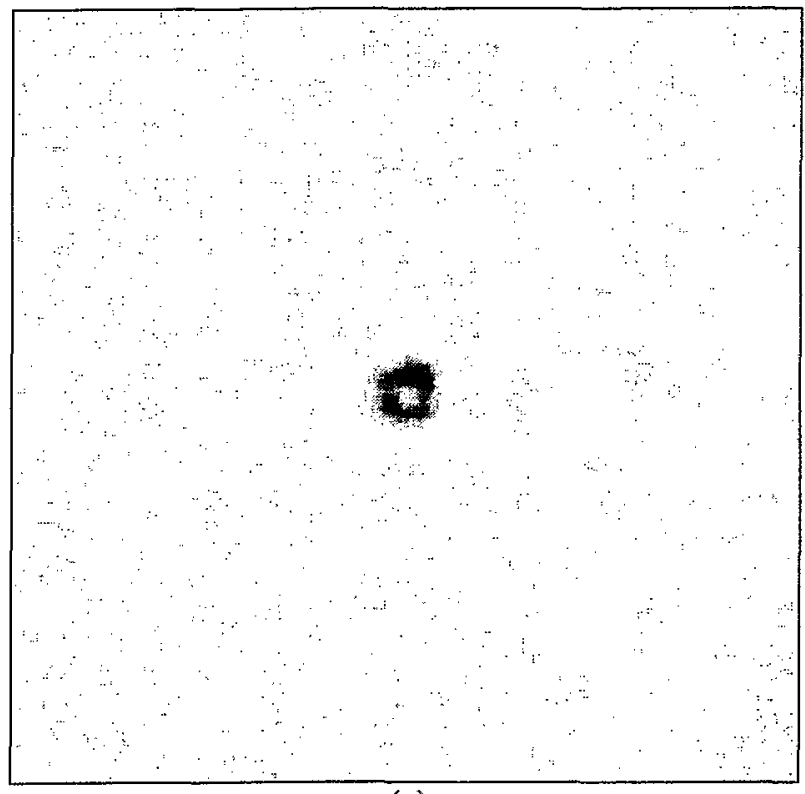

(a)

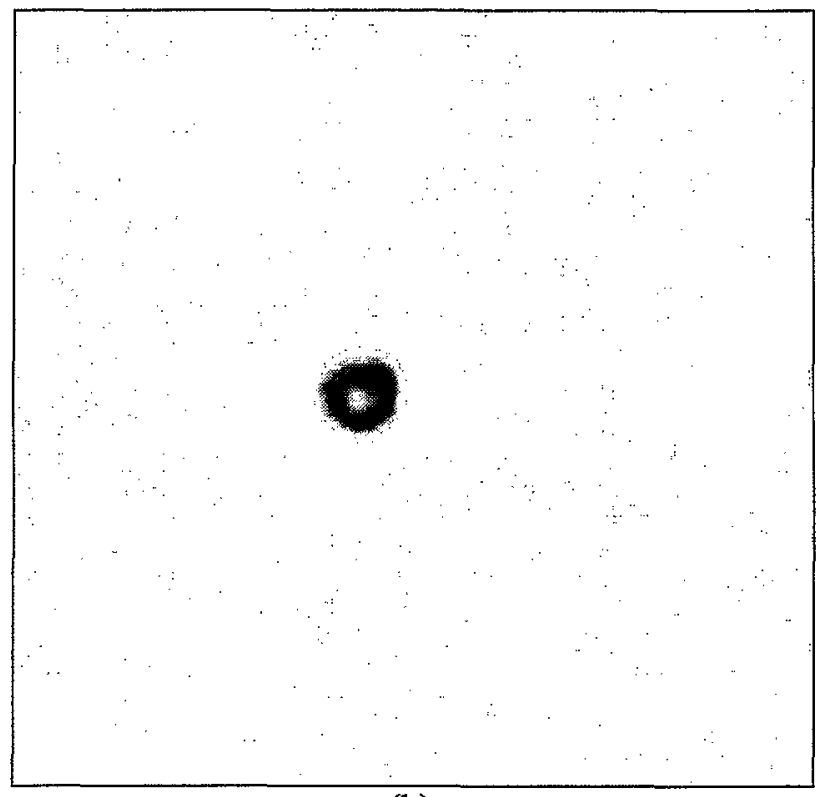

(b)

Figure 7: Images of the end of a plugged paraffin cylinder surrounding the neutron source, framed by (a) a square border of cadmium, and (b) the circular borated plastic shield.

\section{CONCLUSIONS}

The BNL-developed thermal neutron imaging system has been rebuilt with a number of improved components. The precision fabrication of the MURA coded aperture masks and their supporting hardware has allowed better exploitation of the inherently high resolution of the He-3 position-sensitive detector. Faster processing of the raw data using FFTs and a higher speed desktop computer help to overcome previous limitations in image processing, including corrections for the periodic spatial variations in the detector response, and decoding of data on the scale of the detector resolution, rather than the mask pixel. Future developments of this technology will probably involve the use of larger detectors that can collect more neutrons in a given acquisition time. 


\section{ACKNOWLEDGEMENTS}

The author is grateful to Leon Forman for many useful discussions, starting from his original concept of the thermal neutron source locator and the subsequent development and testing of the earlier prototypes. Also, Graham Smith, Neil Schaknowski and Joseph Mead have been extremely helpful in providing and upgrading the position-sensitive neutron detector and electronics. The camera enclosure and mask-supporting structure were constructed by Charles Finfrock, and the Cd masks were fabricated by Max Levy Autograph, Inc. This work has been performed under the auspices of the U.S. Department of Energy Contract No. DE-AC02-98CH10886.

\section{REFERENCES}

1. V. Radeka, N.A. Schaknowski, G.C. Smith and B.Yu, "High Precision Thermal Neutron Detectors", http://www.inst.bnl.gov/GasDetectorLab/NeutronDetectors/hptnd.html, see also_R.A. Boie, J. Fischer, Y. Inagaki, F.C. Merritt, H. Okuno and V. Radeka, "Two-Dimensional High Precision Thermal Neutron Detector, "Nuclear Instruments and Methods, 200, 533-545, 1982.

2. Paul Carlisle, "Coded Aperture Imaging", http://www.ameritech.net/users/paulcarlisle/codedaperture.htmI

3. E.E. Fenimore and T.M. Cannon, "Coded Aperture Imaging with Uniformly Redundant Arrays," Applied Optics, 17, 337-347, 1978.

4. S.R. Gottesman and E.E. Fenimore, "New family of binary arrays for coded aperture imaging", Applied Optics, 28, 4344-52, 1989.

5. K.P. Ziock, L. Nakae, "A Large-Area PSPMT Based Gamma-ray Imager with Edge Reclamation," IEEE Trans. Nuclear Science, 49, 1552-1559, 2002.

6. P. E. Vanier, L. Forman, and E. C. Selcow, "A Thermal Neutron Source Imager Using Coded Apertures, " Proc. Institute of Nuclear Materials Management, 36th Annual Meeting, Palm Desert, CA, 24, pp. 842-847, 1995.

7. Vanier, P. E., and Forman, L., "Advances in Imaging with Thermal Neutrons", Proceedings of the Institute of Nuclear Materials Management, 37th Annual Meeting, Naples, FL, 1996.

8. P.E.Vanier and L. Forman, "Forming Images with Thermal Neutrons", Invited Paper at the SPIE International Symposium on Optical Science and Technology, Conference 4784A, Hard X-rays, Gamma Rays and Particles, Seattle, WA, July, 2002.

9. E.E. Fenimore and T.M. Cannon, "Uniformly redundant arrays: digital reconstruction methods", Applied Optics, 20, 1858-64, 1981. 